\title{
Estadía abreviada poscirugía bariátrica: una posibilidad real sin incrementar complicaciones ni readmisión de pacientes
}

\author{
Percy Brante Báez ${ }^{1}$, Juan Eduardo Contreras Parraguez y Katrina María Lolas Tornquist ${ }^{2}$
}

\begin{abstract}
Abbreviated Hospitalization after bariatric surgery
Introduction: New techniques in bariatric surgery have allowed to reduce complications and surgical mortality. These improvements have made it possible to reduce the hospital stay and accelerate the recovery process, allowing a potential early discharge protocol. Aim: To describe the experience in the use of a protocol designed for an abbreviated stay, in post-operated bariatric surgery patients. Materials and Method: Analytical, retrospective cohort study. A cohort of patients who underwent bariatric surgery, at the Santa María Clinic, by a single surgeon, between July 2014 and April 2019 and submitted to a designed early discharge protocol was considered. Morbidity, mortality, readmissions, and reoperations were recorded in the established period of time. Results: A total of 775 patients were included, 654 patients undergoing laparoscopic sleeve gastrectomy (GEM) and 101 patients undergoing Roux-en-Y gastric bypass (RYGB). There were 8 complications, with no recorded mortality. The readmission/reoperation rate was $4.13 \% / 2.27 \%$ for GEM and $4.55 \% / 3.53 \%$ for RYGB. Conclusions: In this group of patients we were able to demonstrate a low rate of complications, which supports the idea that an early discharge protocol in patients undergoing bariatric surgery is a feasible and safe alternative.
\end{abstract}

Key words: bariatric surgery; laparoscopic sleeve gastrectomy; Roux-en-Y gastric bypass; post operative complications; abbreviated stay.

\section{Resumen}

Introducción: Nuevas técnicas en cirugía bariátrica han permitido disminuir las complicaciones y la mortalidad quirúrgica. Estas mejoras han hecho posible disminuir la estadía hospitalaria y acelerar el proceso de recuperación permitiendo un potencial protocolo de alta temprana. Objetivos: Describir la experiencia en la utilización de un protocolo diseñado de estadía abreviada, en pacientes posoperados de cirugía bariátrica. Materiales y Método: Estudio analítico, retrospectivo de cohorte. Se consideró una cohorte de pacientes en que se realizó cirugía bariátrica, en Clínica Santa María, por un solo cirujano, entre julio de 2014 y abril de 2019, sometidos a un protocolo diseñado de alta temprana. Fueron registradas la morbilidad, la mortalidad, las readmisiones y reintervenciones en el periodo de tiempo registrado. Resultados: Se incluyeron un total de 775 pacientes, 654 pacientes sometidos a gastrectomía en manga laparoscópica (GEM) y 101 pacientes sometidos a bypass gástrico en Y de Roux (RYGB). Hubo 8 complicaciones, sin mortalidad registrada. La tasa de readmisión/reintervención fue de 4,13\%/2,27\% para GEM y 4,55\%/3,53\% para RYGB. Conclusiones: En este grupo de pacientes pudimos demostrar una baja tasa de complicaciones, lo que apoya la idea de que un protocolo de alta temprana en pacientes sometidos a cirugía bariátrica es una alternativa factible y segura.

Palabras clave: cirugía bariátrica; gastrectomía en manga laparoscópica; bypass gástrico en Y de Roux; complicaciones posoperatorias; estadía abreviada.

\section{Introducción}

La cirugía bariátrica es en la actualidad el tratamiento más efectivo para pacientes con obesidad severa. A lo largo de las últimas décadas, la intro- ducción de la técnica laparoscópica y la mejora de diferentes dispositivos han contribuido a reducir significativamente las complicaciones quirúrgicas y la mortalidad. Esto ha permitido acelerar el período de recuperación y acortar la estadía hospitalaria. La
Servicio de Cirugía Clínica Santa María.

2Servicio de Cirugía Hospital San Juan de Dios. Santiago, Chile.

Recibido 2020-08-23, aceptado 2020-11-26

Correspondencia a: Dr. Percy Brante Báez pbrante@clinicasantamaria.cl 
estadía hospitalaria reportada después de la cirugía bariátrica tiene un promedio de entre 2 y 4 días, teniendo una estadía más prolongada si presentan alguna complicación ${ }^{1}$. Existen centros que ya cuentan con programas de alta temprana para pacientes sometidos a cirugía bariátrica ${ }^{2,3}$.

\section{Objetivos}

Describir la experiencia en nuestro centro (Clínica Santa María), en la utilización de un protocolo diseñado de estadía abreviada, en paciente posoperados de cirugía bariátrica.

Establecer variables para poder objetivar la seguridad y eventuales ventajas del uso del protocolo de estadía abreviada en relación con el de alta tradicional.

\section{Materiales y Método}

\section{Población de estudio}

Se incluyeron en el estudio todos aquellos pacientes intervenidos con gastrectomía en manga laparoscópica (GEM) y bypass gástrico laparoscópico en Y de Roux (RYGB), operados por un solo cirujano del Departamento de Cirugía Bariátrica de la Clínica Santa María en Santiago de Chile, en el periodo comprendido entre julio de 2014 y abril 2019, sometidos a un protocolo diseñado de alta precoz posoperatoria.

Se excluyeron de este estudio aquellos pacientes que rechazaron la opción del alta precoz, prefiriendo permanecer en el hospital un día más, pacientes que fueron sometidos a más de una cirugía en el momento de la intervención (vesícula, hernia, etc.) y pacientes reintervenidos por fracaso o conversión de su cirugía previa.

\section{Comité de ética}

Este protocolo fue debidamente presentado y autorizado por el Comité de Ética de Clínica Santa María y cuenta con el respectivo consentimiento informado para su realización.

\section{Variables estudiadas}

Los parámetros evaluados fueron complicaciones relacionadas con cirugía bariátrica, (tromboembolismo pulmonar, trombosis mesentérica, fistula, sangrado, colecciones, etc.), mortalidad, reingresos $\mathrm{y}$ reoperaciones.

Se incluyeron como covariables la edad (en años), el sexo, el tipo de cirugía (GEM y RYGB) y el índice de masa corporal (IMC).

\section{Protocolo estadía abreviada}

Todos los pacientes ingresaron el mismo día de la cirugía. Cuatro horas después de la cirugía se les ofreció iniciar una dieta con agua $(50 \mathrm{cc} / \mathrm{h})$, a tolerancia. Todos los pacientes fueron dados de alta entre las 18 y las $25 \mathrm{~h}$ del posoperatorio con dieta líquida.

La cirugía se realizó bajo anestesia general bajo BIS (índice biespectral) entre 40 y $60^{4}$. El manejo analgésico se realizó mediante bomba de infusión continua con Ketoprofeno (300 mg) y Metamizol (3 g) a las $12 \mathrm{~h}$. En el momento del alta, los pacientes cambian a Ketoprofeno oral asociado a procinéticos durante 5 días. Se utilizaron inhibidores de la bomba de protones (Esomeprazol $40 \mathrm{mg}$ / día) durante 14 días y ácido acetilsalicílico (100 $\mathrm{mg}$ /día) durante 30 días después de la cirugía. El horario de alimentación continúa en casa con dieta de agua $(50 \mathrm{cc} / \mathrm{h})$ durante $24 \mathrm{~h}$, y luego continúa con el régimen de líquidos fraccionados $(150 \mathrm{cc} / \mathrm{h}-600$ calorías) durante 14 días.

Los pacientes fueron monitoreados a las $48 \mathrm{hr}$ tras el alta mediante entrevista telefónica valorando el puntaje del dolor, según la Escala de Evaluación del Dolor (EVA), la medición de la frecuencia cardíaca y la presencia o ausencia de fiebre. El seguimiento incluye una primera cita el día 14 después de la cirugía.

\section{Análisis estadístico}

Este es un estudio analítico, retrospectivo de cohorte.

El análisis estadístico fue realizado con el programa STATA/IC 15.0 (StataCorp, College Station, Texas USA). Las variables cuantitativas fueron descritas como medias y desviaciones estándar y las categóricas como frecuencia absoluta y porcentajes.

\section{Resultados}

Un total de 755 pacientes fueron incluidos en el estudio, 654 pacientes fueron sometidos a una GEM y 101 fueron a RYGB. 345 (45,7\%) hombres y 410 $(54,3 \%)$ mujeres, con una edad media de $38 \pm 11,06$ años (rango 16-71), media de IMC de $36 \pm 5,27$.

Hubo 8 complicaciones $(1,06 \%)$ detectadas dentro de los 30 días posoperatorios y ninguna muerte registrada, lo que se encuentra dentro de las cifras esperadas para estas técnicas ${ }^{5,6}$. En cuanto a las complicaciones: 1 hematoma infectado que fue drenado por punción bajo rayos en una manga gástrica, 1 absceso de tracto de trocar drenado localmente, 3 fístulas, dos en mangas gástricas y una en bypass 
gástrico manejadas endoscópicamente con un stent, 1 tromboembolismo pulmonar subsegmentario, 1 hemoperitoneo laminar, 1 trombosis mesentérica segmentaria, los tres últimos mencionados fueron tratados médicamente. La tasa de readmisión/reintervención fue del 4,13\%/2,27\% para la gastrectomía en manga y del 4,55\%/3,53\% para el bypass gástrico, lo que también se encuentra dentro de los rangos esperados según se describe en la literatura ${ }^{7}$.

\section{Discusión}

Debido a la implementación de la cirugía laparoscópica, asociada a la mejora en los cuidados preoperatorios, se ha logrado reducir la morbimortalidad y la estancia hospitalaria en pacientes sometidos a cirugía bariátrica. En 1997, la mediana de la estadía por cirugía bariátrica (incluyendo RYGB y gastroplastía vertical reforzada o VBG) era de 4 días con una tasa de mortalidad del $0,5 \%$. Ahora las tasas de mortalidad general asociadas a la gastrectomía en manga laparoscópica van de $0,11 \%$ a los 30 días a $0,21 \%$ por año, y para el bypass gástrico es de 0,14 y $0,34 \%$ respectivamente, con una estadía hospitalaria media de 2 días 9.

Actualmente existen varios estudios que utilizan diferentes protocolos de alta precoz en pacientes sometidos a cirugía bariátrica ${ }^{10,11}$.

Con base en estos resultados, creemos que la implementación de nuestro protocolo de alta tem- prana parece ser seguro y efectivo para el grupo de pacientes seleccionados.

Las limitaciones de este estudio incluyen su diseño de cohorte retrospectivo. También es importante mencionar que la falta de un grupo de control nos impide llegar a conclusiones más categóricas, a pesar de que los datos registrados están en relación con los reportados en la literatura.

\section{Conclusión}

Con base en nuestros resultados, concluimos que la implementación de un protocolo de estadía abreviada o alta precoz en cirugía bariátrica podría ser una alternativa factible y segura en el manejo estos pacientes. La implementación de este protocolo, además, demuestra una baja tasa de reingresos, reintervenciones, complicaciones mayores y mortalidad, lo que apoya nuestra idea.

\section{Responsabilidades éticas}

Protección de personas y animales. Los autores declaran que para esta investigación no se han realizado experimentos en seres humanos ni en animales.

Confidencialidad de los datos. Los autores declaran que en este artículo no aparecen datos de pacientes.

Conflictos de interés: no hay.

\section{Bibliografía}

1. Schauer P. Outcomes After Laparoscopic Roux-en-Y Gastric Bypass for Morbid Obesity. Ann Surg. 2000;232:515-29.

2. McCarty TM, Arnold DT, Lamont JP, Fisher TL. Optimizing outcomes in bariatric surgery: outpatient laparoscopic gastric bypass. Ann Surg. 2005;242:494501.

3. Geubels N, Bruin SC, Acherman YI, Van de Laar AW, Hoen MB, De Brauw LM. Fast track care for gastric bypass patients decreases length of stay without increasing complications in an unselected patient cohort. Obes Surg. 2014;24:390-6.

4. Buisán F, Ruiz N; Grupo de Trabajo de la Sociedad Castellano-Leonesa de Anestesiología, Reanimación y
Terapéutica del Dolor (SOCLARTD). Índice biespectral (BIS) para monitorización de la consciencia en anestesia y cuidados críticos: guía de práctica clínica. Valladolid: SOCLARTD; 2008.

5. Hutter MM, Schirmer BD, Jones DB, Ko CY, Cohen ME, Merkow RP, et al and On behalf of the ACS-BSCN Advisory Committee. First Report from the American College of Surgeons-Bariatric Surgery Center Network: Laparoscopic Sleeve Gastrectomy has Morbidity and Effectiveness Positioned Between the Band and the Bypass. Ann Surg. 2011;254:410-22.

6. Lancaster RT, Hutter MM. Bands and bypasses: 30 -day morbidity and mortality of bariatric surgical procedures as assessed by prospective, multi-center, risk-adjusted ACS-NSQIP data. Surg Endosc. 2008;22:2554.

7. Saunders JK, Ballantyne GH, Belsley S, Stephens D, Trivedi A, Ewinget DR, et al. 30-day readmission rates at a high volume bariatric surgery center: laparoscopic adjustable gastric banding, laparoscopic gastric bypass, and vertical banded gastroplasty-Roux-en-Y gastric bypass. Obes Surg. 2007;17:1171-7.

8. Berger ER, Huffman KM, Fraker T, Petrick AT, Brethauer SA, Hall BL, et al. Prevalence and Risk Factors for Bariatric Surgery Readmissions: Findings From 130,007 Admissions in the Metabolic and Bariatric Surgery Accreditation and Quality Improvement Program. Ann Surg. 2018;267:122-31.

9. Mahmood M, Sharples A, Rotundo A, Balaji N, Rao V. Factors Predicting Length of Stay Following Bariatric 


\section{ARTÍCULO ORIGINAL}

Surgery: Retrospective Review of a Single UK Tertiary Centre Experience. Obes Surg. 2018;28:1924-30.

10. Lemanu DP, Singh PP, Berridge K, Burr M, Birch C, Baboret R, al.
Randomized clinical trial of enhanced recovery versus standard care after laparoscopic sleeve gastrectomy. Br J Surg. 2013;100:482-9.

11. Awad S, Carter S, Purkayastha S,
Hakky S, Moorthy K, Cousinset J, et al. Enhanced recovery after bariatric surgery (ERABS): clinical outcomes from a tertiary referral bariatric centre. Obes Surg. 2014;24:753-8. 